\title{
Cellular Flow in Paper-based Microfluidics
}

Gihoon Choi and Seokheun Choi*

Bioelectronics \& Microsystems Lab, Department of Electrical \& Computer Engineering, State University of New York-Binghamton, Binghamton, New York, 13902, USA

We reported a novel, non-optical technique to analyse and quantify the transportation of bacterial cells in paper-based microfluidics. This approach was based on real-time measurement of the microbial electricity production. Our device was designed to have three hydrophilic regions linked by a channel on a paper layer by patterning hydrophobic barriers in the paper. Each region came into contact with an anodic electrode layer which was stacked with a cathodic compartment through a proton exchange membrane. When bacterial cells were transported to each region by capillary force, the bacteria cells were promoted to adhere onto the anode and the cells completed their respiration by transferring the electrons to the anode. A conductive load connected the anode and cathode to complete the external circuit through which the electrons flowed. The protons generated by the anodic reactions passed through the proton exchange membrane and travelled to the cathode, where they combined with electrons in the reduction process. By measuring the current through the load, bacterial cells' transportation through the channels in paper was quantitatively investigated according to the pore size of the paper. Further understanding was made by analysing electron microscope examination of the hydrophilic regions. This work will determine more efficient cell movement within a paper material by controlling the paper's microfluidic dimensions and pore size. It will also provide a quantitative understanding of the flow of large organisms for use in paper-based diagnostics.

\section{INTRODUCTION}

Paper-based microfluidics have attracted significant research interest and gained acceptance as a potential technique for low-cost point-of-care diagnostic sensors. ${ }^{1,2}$ These paper microfluidic devices have a distinct advantage in that no pump/tubing is required for liquid transport, thus no external power source is needed. ${ }^{3-5}$ Many paper-based microfluidics are already available for the detection of a wide spectrum of biomolecules with minimal expertise and external equipment. ${ }^{6-11}$ Paper-based microfluidics are focused on analyte delivery to the detection zone where sophisticated sensing processes are performed. Liquid transportation in paper-based microfluidics occurs via passive capillary action and the transport of homogenous liquid samples has been well explored in recent literature. ${ }^{12-15}$ However, the flow information of a heterogeneous sample such as cells and particles, (e.g. whole blood, urine, etc.) is not yet fully 
understood because monitoring the flow in a non-transparent and porous paper matrix remains a challenge, rendering the traditional particle-imaging velocimetry unsuitable. ${ }^{16}$ Thus, there is an urgent need for a novel, non-optical technique to analyze and quantify the particles' flow in a paper matrix.

In this work, we created a new analytical tool to study the transportation of bacterial cells in paper for better understanding of the flow of particles in a paper matrix. We leveraged the techniques recently demonstrated for a paper-based microbial fuel cell (MFC). ${ }^{17-22}$ A paper anode reservoir allows for rapid adsorption of bacteria-containing liquid. This adsorption immediately promotes bacteria cell attachment to the electrode, where bacterial respiration can then transfer electrons from the organic liquid to the electrode. Therefore, paper-based MFCs show a very short start-up time relative to conventional MFCs made of the usual rigid materials. ${ }^{23}$ This prominent feature has motivated us to develop an analytical tool for monitoring the flow of bacterial cells in a paper matrix. Our device contained three hydrophilic spots linked by a hydrophilic channel on paper. Each spot came into contact with an anode that was electrically connected to a cathode through an external load. When bacterial cells were transported to each spot by capillary force, the current generated from the bacteria was monitored and bacterial cells' transportation through the channel was quantitatively investigated according to the pore size of the paper. This work will provide a quantitative understanding of the bacterial cells for the use in paper-based microfluidics.

\section{Materials and Methods}

\section{A. Device Fabrication and test setup}

The paper-based microfluidic device consisted of five functional layers: an anode layer, an anodic paper chamber (Whatman \#4 or \#5), a proton exchange membrane (PEM), a cathodic paper chamber (Whatman \#1), and a cathode layer (Fig. 2 \& Fig. 3). Anodic and cathodic paper chambers featuring hydrophilic zones with hydrophobic wax boundaries were microfabricated by heat pressing commercially available wax paper onto filter paper. We used a screen-printed carbon anode on the polymethly methacrylate (PMMA) substrates. All layers were carefully aligned and clamped with 5 screws (Fig. 3). The paper-based microfluidic device contained two sets of three hydrophilic spots linked through microfluidic channels (Fig. 2 \& Fig. 3). Each spot came into contact with an anode electrically connected to a cathode through an external load. The current generated from electrogenic bacterial cells were measured on each spot (Fig. 1). We measured the potential between the anode and the cathode with a data acquisition system (National Instrument, USB-6212) and recorded the results every 1 min via a customized LabVIEW interface. ${ }^{17,18}$ An external resistor was connected between 
the electrodes of the device to close the circuit. We calculated current through the external resistor via Ohm's law and the output power. We tested two different paper substrates with different pore sizes: Whatman \#5 (pore size: $2.5 \mu \mathrm{m}$ ) and \#4 (pore size: $20 \mu \mathrm{m}$ ) (Table 1).

\section{B. Anolyte and catholyte}

Wile-type Shewanella oneidensis MR1 (1 3 $\mu \mathrm{m}$ in length and $\sim 1 \mu \mathrm{m}$ in width) were grown in L-broth medium under the consistent temperature at $30{ }^{\circ} \mathrm{C}$ and utilized as a bacterial model for studying bacterial cells' transportation in the paper matrix. L-broth medium contained $10.0 \mathrm{~g}$ triptone, $5.0 \mathrm{~g}$ yeast extract and $5.0 \mathrm{~g} \mathrm{NaCl}$ per $1 \mathrm{~L}$ of distilled water. Bacterial cells were harvested by centrifugation and re-suspended in a new L-broth medium. The catholyte was $50 \mathrm{mM}$ ferricyanide in a $100 \mathrm{mM}$ phosphate buffer in which the $\mathrm{pH}$ was adjusted to $7.5 \pm 0.2$ with $0.1 \mathrm{M} \mathrm{NaOH}$.

\section{Bacterial fixation and SEM imaging}

The device was disassembled and rinsed after the current measurement. The anodic paper reservoir was gently soaked into $2 \%$ glutaraldehyde solution for 24 hours at room temperature. The bacteria in porous structural paper immediately fixed onto the substrate. Then, the samples were dehydrated by serial 5 min transfers through $25,50,70,80,90,95$, and $100 \%$ ethanol. The dehydrated samples were thoroughly dried by nitrogen gas, and mounted on specimen stub with adhesive carbon tape. Roughly 10-20nm thick gold/palladium was coated to minimize possible charging effect while taking images. The fixed bacteria strains were investigated using a FESEM (Field Emission SEM) (Supra 55 VP, Zeiss).

\section{RESULTS AND DISCUSSION}

\section{A. Bacterial transportation and current generation}

As shown in Fig. 2, when a Shewanella sp. inoculum was dropped on spot \#1, many bacterial cells stay in the reservoir of spot \#1 while some bacterial cells are transported to spot \#2 and then spot \#3 by capillary force. Once they arrive on each spot and attach on the anodes, the cells transfer electrons to each electrode through their metabolism and protons generated from this reaction pass from the anodic reservoir to the cathode through the PEM. A conductive load connects the two electrodes to complete the external circuit, enabling the current flow.

Fig. 3 shows current profiles measured from each spot according to the pore size of the paper reservoir (Whatman \#5: 2.5 $\mu \mathrm{m}$ and Whatman \#4: $\sim 20 \mu \mathrm{m}$ ). The control media sample 
without bacteria produced a certain amount of current mostly from spot \#1 because chemical ions present in the media contributed the current generation. As the ions in spot \#1 were depleted and the media travelled toward spot \#3, the value at spot \#1 reached almost zero at 13 min in Whatman \#5 and 5 min in Whatman \#4, respectively. Whatman \#5 with smaller pore size required more time to use up the ions. However, the currents generated from controls were much smaller than the samples' ones, indicating that the major current source from the samples was bacterial metabolism.

When the bacterial samples were dropped on spot $\# 1 \mathrm{~s}$, the transient currents were measured on both paper microfluidic devices. These instant peak currents were generated from the ions in media (like controls' current generation), which travels faster than the bacterial cells. The transient currents rapidly dropped within a minute but the overall current values increased again due to the currents generated from the bacterial cells. The current on spot \#1 in Whatman \#5 was smaller than that in Whatman \#4. This is because the cells in Whatman \#5 could not travel well through the smaller pores and reach the anodic surface. In both filter papers, the measured currents from spot \#2 were about $2 \mathrm{x}$ smaller than that from spot \#1 because the cell numbers decreased significantly passing through the paper matrix. We then measured a much smaller value of current from spot \#3. While it was difficult for bacterial cells to pass through smaller pore-sized paper, larger pore-sized paper allowed more bacterial cells to be transported a greater distance within a shorter time period. It is notable that there is a dropping point in the current profile of Whatman \#4 while Whatman \#5 shows gradual increase. This is probably because the quickly attached cells through the larger pores voraciously start their metabolic processes and rapidly deplete the organic matter. On the contrary, in Whatman \#5 with the smaller pores, low metabolism and poor electron transfer efficiency from the smaller number of cells contribute to the longer but smaller current generation.

\section{B. SEM images}

Bacterial cells' transportation in the paper matrix can be further explained by analysing electron microscope examinations of the paper microfluidic layer (Fig. 4). For Whatman \#5, a large number of cells were observed on spot \#1 while a less number of cells were found on spot \#2 as the sample proceeded through the microfluidic channel. On spot \#3, we could barely see the cells. However, Whatman \#4 showed a much less number of cells on spot \#1 than those on Whatman \#5 while spot \#2 of Whatman \#4 had as many as the total number of cells on spot\#1 of Whatman \#4. These phenomena can be explained with Fig. 5. The small pore-sized paper (Whatman \#5) allowed less number of bacterial cells to be transported and many of the cells remained around the inlet of the spot \#1 while the large pore-sized paper 
(Whatman \#4) could equally distribute the cells throughout the channels toward spot \#2 via strong capillary force. However, the spot\#3 was too far from the inlet to move the cells. These results are in a good agreement with the current profiles shown in Fig. 3. The bacteria transport was mainly driven by the strong capillary force in the paper, which promoted the rapid adsorption of bacterial samples, enabling the cell attachment to the anode. Bacterial movement in a paper matrix can be effectively controlled by the paper's pore size and its hydrophilic reservoir volume.

\section{Quantitative investigation on cells' transportation}

Although the total number of bacterial cells transported through the paper matrix cannot be directly quantified, the transport rate of the cells by the types of papers can be indirectly determined. Given that the bacterial electron transfer mechanisms through the paper matrix are mainly based on direct electron transfer in the experimental setup, ${ }^{17}$ the current generation is proportional to the number of cells. For Whatman \#5 (pore size: $2.5 \mu \mathrm{m}$ ), $2.23 \mu \mathrm{A}$ on spot \#1 decreased to $0.90 \mu \mathrm{A}$ on spot $\# 2$ and $0.37 \mu \mathrm{A}$ on spot $\# 1$ while the cells were transported through the $4 \mathrm{~mm}$-long ( $2 \mathrm{~mm}$ wide and $200 \mu \mathrm{m}$ thick) microfluidic paper channels, which corresponds to around $40 \%$ of the transport rate for both channels. On the other hand, Whatman \#4 (pore size: $20 \mu \mathrm{m}$ ) provided $56 \%$ and $29 \%$ of the rate through channel 1 (spot \# 1 to spot \#2) and channel 2 (spot \#2 to spot \#3), respectively. The small pore size paper offered the more consistent transport rate for the cells being flowed to spot \#3 while the larger pore size paper had higher transport rate for the first channel but significant drop for the further distance.

\section{Potential future applications}

Despite their vast potential, the paper-based microfluidics are limited to the homogenous liquid samples (e.g. plasma) and still suffers from the difficulties in transporting particles, cells, and large biomolecules in the paper, decreasing effectiveness of clinical assays due to bimolecular interaction with paper fibers. Given that the deadly diseases in the developing countries, where the paper-based microfluidic device is the most promising diagnostic platform, are infectious ones such as HIV/AIDS, malaria and measles, ${ }^{24-28}$ there is an urgent need to understand the movement of large biomolecules (e.g. bacterial cells) within a paper material. This new technique described in this report will provide a quantitative understanding of the flow of different sizes of bacterial cells in various types of paper materials. ${ }^{29}$ Also, other types of large biomolecules' movement in the paper can be simulated and anticipated to develop novel biosensing assays for infectious diseases. With an in-depth understanding of the particle's transportation in paper, we will be able to (i) improve the effectiveness of paper 
assays by decreasing interference of large-sized biosamples, (ii) provide a new strategy for delivering large particles (e.g. cells) in paper-based microfluidics and (iii) take better advantage of the sizeexclusion properties of paper to remove particles from whole blood.

\section{Conclusion}

We developed a superior analytical tool to investigate the bacterial transport behavior in paper for a better understanding of the flow of particles in a porous matrix. This technique was based on measuring, in real time, the electricity generated from bacteria. Although paper-based microfluidic devices have attracted considerable interest in numerous applications including clinical diagnosis, food quality control, and environmental monitoring, the devices still suffer from difficulties in transporting particles or large organisms in the paper matrix, and to date, no research groups have made efforts to explore the transportation of bacterial cells through the paper matrix. Here, we monitored the flow of bacterial cells in two different paper substrates. This work will realize more efficient particle movements within a paper layer by controlling the paper's microfluidic dimensions and pore size. It will also provide a quantitative understanding of the flow of large organisms for the use in paper-based diagnostics.

\section{Acknowledgment}

This work is supported by NSF (ECCS \#1503462). We would like to thank the Analytical and Diagnostic Laboratory at SUNY-Binghamton for providing the facilities.

\section{REFERENCES}

1 D.D. Liana, B. Raguse, J.J. Gooding, and E. Chow, Sensors, 2012, 12, 11505-11526.

2 A.K. Yetisen, M.S. Akram, and C.R. Lowe, Lab Chip, 2013, 13, 2210-2251.

3 T.H. Nguyen, A. Fraiwan and S. Choi, Biosensors and Bioelectronics, 2014, 54, 640-649.

4. Y. Zhang, C. Zhou, J. Nie, S. Le, Q. Qin, F. Liu, Y. Li, and J. Li, Anal Chem, 2014, 86, 2005-2012.

5. A.W. Martinez, S.T. Phillips, E. Carrilho, S.W. Thomas III, H. Sindi, Anal Chem, 2008, 80, 3699-3707.

6 L. Li, J. Xu, X. Zheng, C. Ma, X. Song, S. Ge, J. Yu, and M. Yan, Biosensors and Bioelectronics, 2014, 61, 76-82.

7 W. Lan, E.J. Maxwell, G. Parolo, D.K. Bwambok, A.B. Subramaniam, and G.M. Whitesides, Lab Chip, 2013, 13, 4103-4108. 
8 C. Cheng, A.W. Martinez, J. Gong, C.R. Mace, S.T. Phillips, E. Carrilho, K.A. Mirica, and G.M. Whitesides, Angew. Chem. Int. Ed., 2010, 49, 4771-4774.

9 H. Liu, Y. Xiang, Y. Lu, and R.M. Crooks, Angew. Chem. Int. Ed., 2012, 51, 6925-6928.

10 J. Hu, S. Wang, L. Wang, F. Li, B. Pingguan-Murphy, T.J. Lu, and F. Xu, Biosensors and Bioelectronics, 2014, 54, 585-597.

11 W.L. Then and G. Garnier, Rev Anal Chem, 2013, 32, 269-294.

12 L. Li, X. Huang, W. Liu, and W. Shen, Appl. Mater. Inter., 2014, 6, 21624-21631.

13E. Fu, S.A. Ramsey, P.Kauffman, B. Lutz, and P. Yager, Microfluid Nanofluidics, 2011, 10, 29-35.

14 X. Wang, J.A. Hagen, and I. Papautsky, Biomicrofluidics, 2013, 7, 014107.

15 B.R. Lutz, P. Trinh, C. Ball, E. Fu, and P. Yager, Lab Chip, 2011, 11, 4274-4278.

16 P. Kauffman, E. Fu, B. Lutz, and P. Yager, Lab Chip, 2010, 10, 2614-2617.

17 H. Lee, and S. Choi, Nano Energy, 2015, 15, 549-557.

18 G. Choi, D. Hassett, and S. Choi, Analyst, 2015, 140, 4277-4283.

19 A. Fraiwan, and S. Choi, Physical Chemistry Chemical Physics, 2014, 16, 26288-26293.

20 A. Fraiwan, H. Lee and S. Choi, IEEE Sensors Journal, 2014, 14, 3385-3390.

21 A. Fraiwan, S. Mukherjee, S. Sundermier, H.-S. Lee and S. Choi, Biosensors and Bioelectronics, 2013, 49, 410-414.

22 G. Choi, and S. Choi, Analyst, 2015, 140, 5901-5907.

23 S. Choi, Biosensors and Bioelectronics, 2015, 69, 8-25.

24 R. McNerney, Diagnostics, 2015, 5, 200-209.

25 A.A. Kumar AA, J.W. Hennek, B.S. Smith, S. Kumar, P. Beattie, S. Jain, J.P. Rolland, T.P. Stossel, C. Chunda-Liyoka, G.M. Whitesides, Angew Chem Int Ed, 2015, 54, 5836-5853.

26 D. Mabey, R.W. Peeling, A. Ustianowski, M.D. Perkins, Nature Reviews Microbiology, 2004, 2, 231-240.

27 X. Mao and T.J. Huang, Lab Chip, 2012, 12, 1412-1416.

28 P. Yager, T. Edwards, E. Fu, K. Helton, K. Nelson, M.R. Tam, B.H. Weigi, Nature, 2006, $442,412-418$.

29 G. Choi and S. Choi, "Bacterial cell transportation in paper-based microfluidics," Transducers 2015, Jun. 21 - 25, 2015, Anchorage, Alaska, USA, pp. 1921-1924 
Figures \& Table
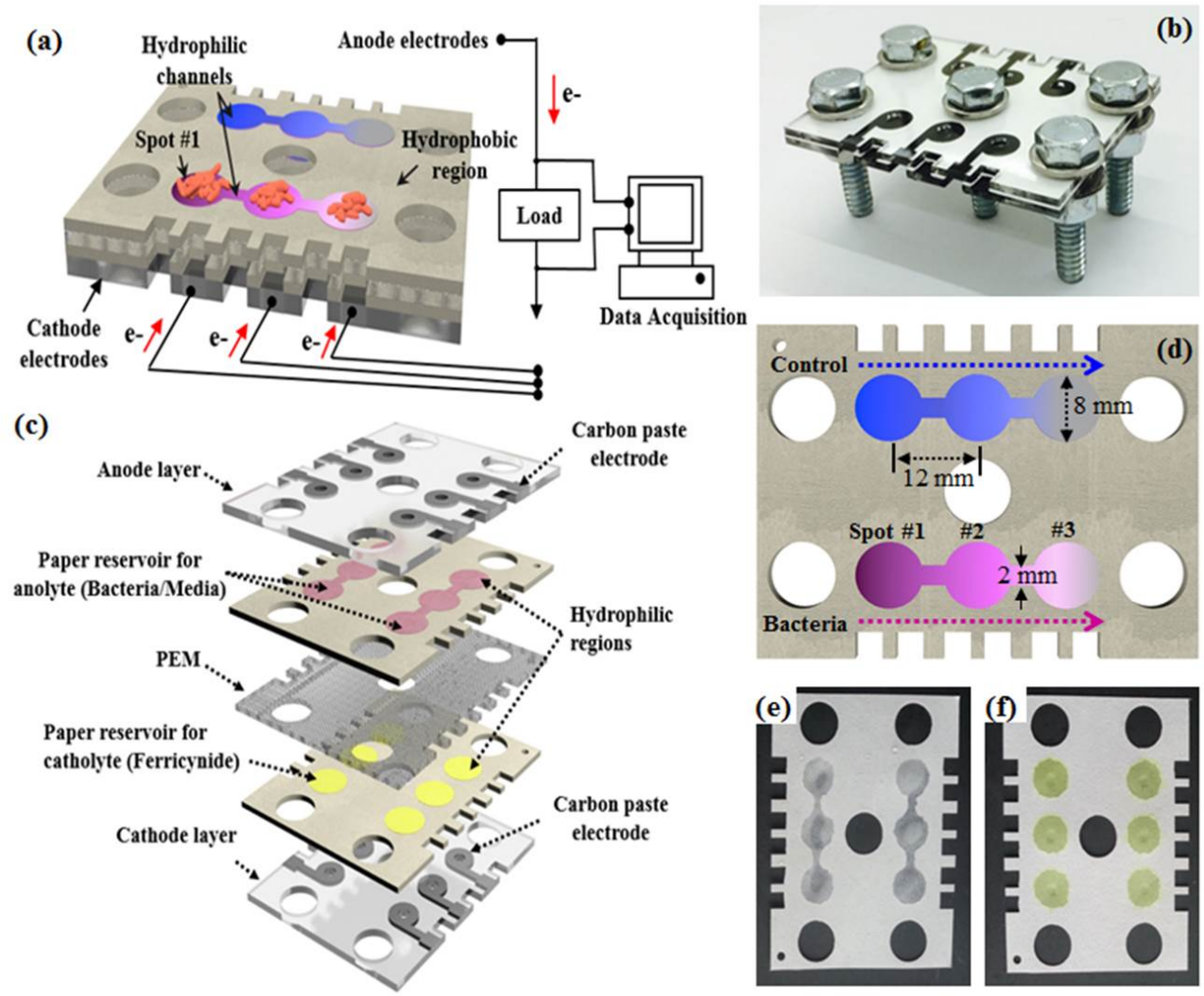

Figure 1. (a) Schematic diagram of the test setup. The bacterial cells were dropped on spot \#1 and transported to spot \#3 via capillary force. Currents can be measured once the cells arrived. The cathodic paper contained only reservoirs without fluidic channels. (b) Photo-images of the assembled device. Schematic diagram of (b) individual layers of the device and (c) the paper reservoir with dimensions. Photo-images of the paper reservoirs after loading the (e) anolyte and (f) catholyte.

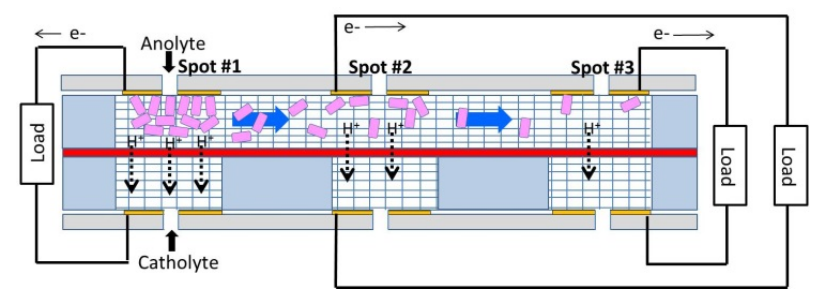

Figure 2. Schematic diagram of the proposed technique for measuring bacterial cells' transportation in a paper matrix. Currents were measured from each spot according to the pore size of the paper reservoir. 

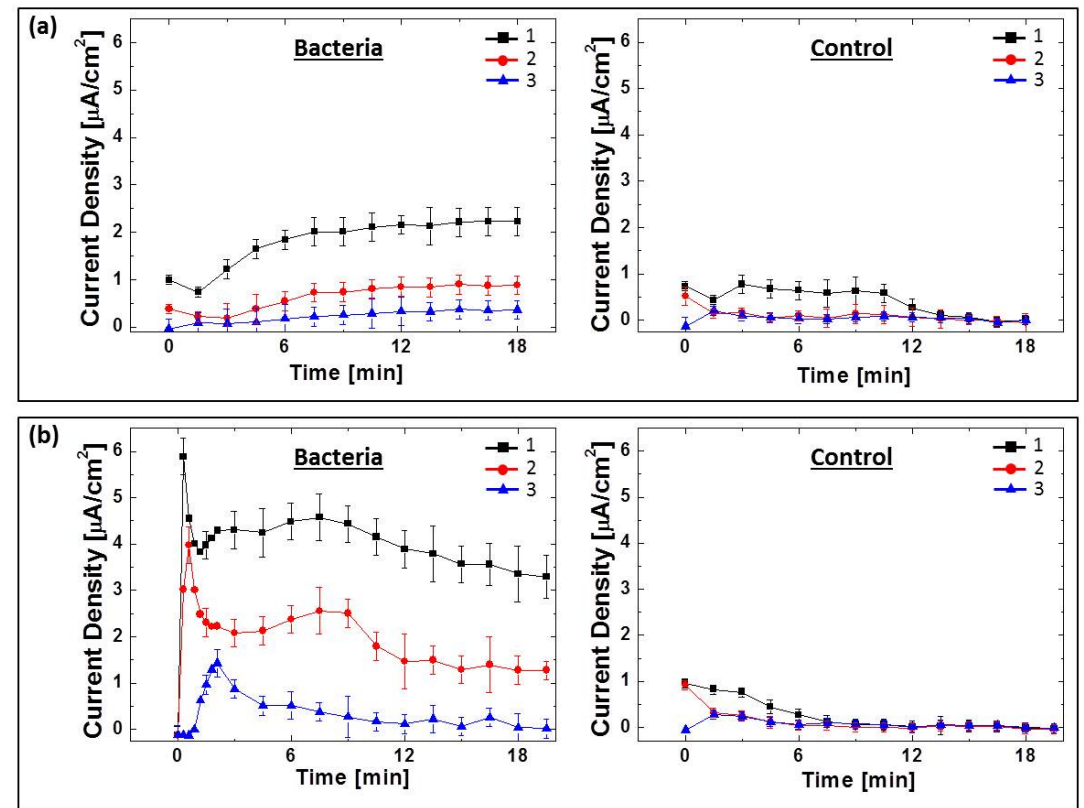

Figure 3. Currents profiles generated from each spot on (a) Whatman \#5 filter paper (2.5 $\mu \mathrm{m}$ pore size) and (b) Whatman \#4 filter paper (20 $\mu \mathrm{m}$ pore size). Shewanella oneidensis was selected as a model microorganism and L-broth media (without bacteria) as a control. The samples were dropped on spot \#1 and current generations on each spot were monitored as they travelled through the channel toward spot \#3. 


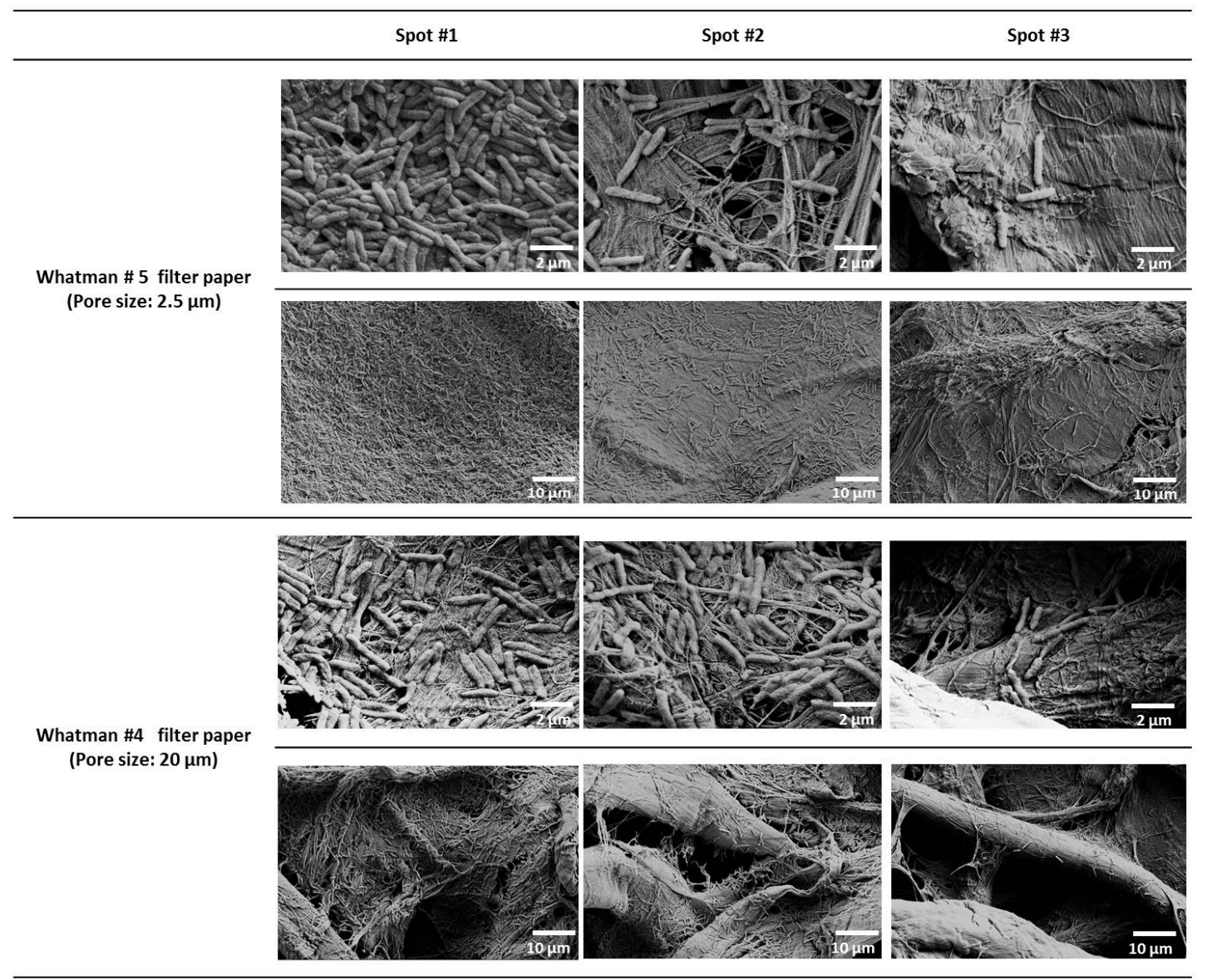

Figure 4. SEM images of each spot (\#1, \#2, \& \#3) on papers with different pore size after all the experiments.

(a)

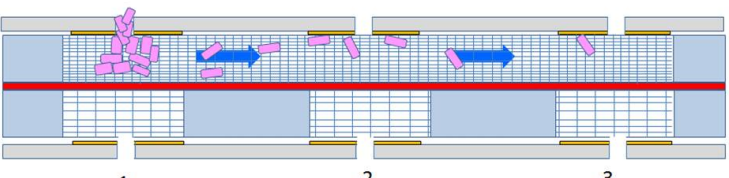

(b)

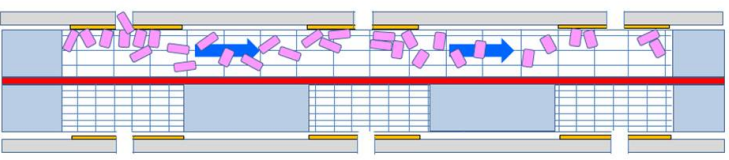

Figure 5: Schematic diagram of the bacterial transportation through different paper substrates with different pore sizes. 
Table1. Types of paper reservoirs used in this work. Two different paper substrates with different specifications were tested for anodic paper chambers.

\begin{tabular}{|c|c|c|}
\hline & $\begin{array}{c}\text { Whatman }^{\circledR} \\
\text { Grade \#5 }\end{array}$ & $\begin{array}{c}\text { Whatman }^{\circledR} \\
\text { Grade \#4 }\end{array}$ \\
\hline Pore size & $\sim 2.5 \mu \mathrm{m}$ & $20 \sim 25 \mu \mathrm{m}$ \\
\hline Porosity & Fine & Coarse \\
\hline Flow rate & $1420 \mathrm{sec} / 100 \mathrm{~mL}$ & $37 \mathrm{sec} / 100 \mathrm{~mL}$ \\
\hline Thickness & $\sim 200 \mu \mathrm{m}$ & $\sim 205 \mu \mathrm{m}$ \\
\hline
\end{tabular}




\section{Biographies}

Gihoon Choi was born in South Korea in 1987. He received the BS \& MS degrees in Electrical and Computer Engineering from State University of New York, Binghamton, in 2013 and 2015, respectively. He is currently working toward the Ph.D degree at Pennsylvania State University.

Seokheun Choi was born in South Korea in 1977. He received the BS \& MS degrees in Electrical engineering from Sungkyunkwan University, Korea, in 2003 and 2004, respectively. After graduating, he was a research engineer at LG Chem, Ltd. from 2004 to 2006. He earned his PhD in Electrical Engineering from Arizona State University in 2011. From 2011 to 2012, he was a research professor in the School of Electric \& Computing Systems at the University of Cincinnati. $\mathrm{He}$ is currently an assistant professor with the Department of Electrical \& Computer Engineering at State University of New York, Binghamton. He has published over 50 journal and conference articles, two book chapters, and one book and he holds two U.S. patents. His areas of interest are BioMEMS/Biosensors/Biofuel Cells. 